\title{
OPERATIONAL CALCULUS AND THE FINITE PART OF DIVERGENT INTEGRALS
}

\author{
BY \\ T. K. BOEHME(1)
}

1. The use of the finite part of divergent integrals started with A. Cauchy $[1 ; 2](2)$, who used what he called an "integrale extraordinaire" to give a sense to the gamma function for negative values of the argument. Since that time the notion has been used and extended by various authors. J. Hadamard [3] extended the concept to multiple integrals. He and F. Bureau [4] have used the finite part of divergent integrals as an important tool in solving partial differential equations. Lately L. Schwartz [5] and M. Lighthill [6] have applied the theory of distributions to extend the idea of the finite part of divergent integrals.

P. L. Butzer [7] used the operational calculus of J. Mikusiński to study the finite part of divergent convolution integrals. He has shown an extension in Mikusiński's operational calculus of the integral $\int_{0}^{t} g(t-u) u^{\alpha} d u$ with $\alpha \geqq-1$ to FP $\int_{0}^{t} g(t-u) u^{\alpha} d u$ with $\alpha$ any real number. This extension, at least in the case when $\alpha$ is not a negative integer, is a very natural one. It is the work of Butzer which is followed up in this paper.

In $\S 2$ a foundation is laid for what is to follow. The concept of an analytic operator function, which will be necessary in $\S 3$, is introduced and the useful concept of the logarithm of an operator is discussed.

The operator function $[\operatorname{FP} f(z, t)]$ which is associated with the function $\{f(z, t)\}$ is defined in $\S 3$, and the relationship of this operator function to

$$
\mathrm{FP} \int_{0}^{t} g(t-u) f(z, u) d u
$$

is discussed when $\{f(z, t)\}$ and $g$ are such that FP $\int_{0}^{1} g(t-u) f(z, u) d u$ is defined. It is found that for many functions $\{f(z, t)\}$ and $g$, the operator product $g[\operatorname{FP} f(z, t)]$ reproduces the finite part of the definite integral. The operator product has the advantage that it exists for all operators $g$, whereas the finite part of the divergent integral exists only if $g$ satisfies certain smoothness conditions. Also, the operator product is defined for certain functions $\{f(z, t)\}$ for which the finite part of the divergent integral has not been defined.

Received by the editors January 15, 1962.

(1) This paper consists of a portion of the author's doctoral thesis offered in partial fulfillment of the requirements for the Doctor of Philosophy degree at California Institute of Technology. While writing the thesis the author was supported financially by California Institute of Technology and the National Science Foundation.

(2) Numbers in brackets refer to the bibliography at the end of the paper. 
In $\S 4$ it is shown how to find the inverse of some of the operators [ $\operatorname{FP} f(z, t)]$ and how to utilize these in solving improper integral equations involving the finite part of divergent convolution integrals on the half-line $t \geqq 0$. A problem posed by Butzer is solved. The problem is to solve the singular integral equation

$$
\operatorname{FP} \int_{0}^{t} f(t-u) \frac{J_{0}(u)}{u} d u=g(t), \quad t>0 .
$$

2.1. Let the interval $t \geqq 0$ be denoted by $I$. The functions to be considered in what follows will unless otherwise noted be functions on $I$ to the complex numbers. With the usual notion of pointwise addition and multiplication by scalars these functions form a vector space over the field of complex numbers. The symbols $f$ and $\{f(t)\}$ will be used to denote an element of this vector space; the function whose value is one for all $t \in I$ will be denoted by $h$, and the function whose value is zero for all $t \in I$ will be denoted by $0 . f(t)$ will denote the value of the function $f$ at the point $t$. The function $|f|$ is related to the function $f$ by the definition $|f|(t)=|f(t)|$ for all $t \in I$. Greek letters $\alpha, \beta, \zeta, \cdots$ will generally denote scalars.

If a function is integrable, bounded, absolutely continuous, etc., on every closed and bounded subinterval of $I$ it will be said to have this property locally.

The space of continuous functions on $I$ will be called $C^{*}$. If $f_{n}, n=1,2, \cdots$ are in $C^{*}$ we say $f_{n} \rightarrow f\left(C^{*}\right)$ as $n \rightarrow \infty$ if the $f_{n}$ converge uniformly to $f$ on each compact subinterval of $I$. The space of locally integrable functions on $I$ will be called $L^{*}$. If $f_{n}, n=1,2, \cdots$ are in $L^{*}$ and $\int_{0}^{K}\left|f(x)-f_{n}(x)\right| d x \rightarrow 0$ as $n \rightarrow \infty$ for for each $K>0$ we will say $f_{n} \rightarrow f\left(L^{*}\right)$ as $n \rightarrow \infty$.

We will give a brief survey of the foundations of Mikusiński's operational calculus. Most of what is in this section can be found in Mikusinski's Operational calculus [8] and also in Erdélyi's Operational calculus and generalized functions [9].

Let $f$ and $g$ be locally integrable. The function defined by

$$
k(t)=\int_{0}^{t} f(t-u) g(u) d u \quad \text { almost all } t \geqq 0
$$

is called the finite convolution of $f$ and $g$. It is very well known that the finite convolution of two locally integrable functions is a locally integrable function, and the finite convolution of two continuous functions is a continuous function. The finite convolution defines a multiplication which makes $L^{*}$ and $C^{*}$ into commutative rings. This multiplication will be denoted by juxtaposition; thus the above equation will be written $k=f g$. It is a corollary to a theorem of Titchmarsh that $L^{*}$ and $C^{*}$ have no divisors of zero. The ring $C^{*}$ can be extended to a field $F$, its quotient field, whose elements are of the form $a / b$, where $a, b \in C^{*}$, and where $b \neq 0$. These elements are called Mikusiński operators or just opera- 
tors. The ring $L^{*}$ is isomorphically imbedded in $F$ under the mapping $f \rightarrow f c / c$ where $f \in L^{*}, c \in C^{*}$, and $c \neq 0$. The field of complex numbers is isomorphically imbedded in $F$ by the mapping $\alpha \rightarrow \alpha \cdot 1$ where 1 is the unit element of $F$. The unit element of $F$ will be written as 1 ; the zero element of $F$, like the function $\{0\}$, will be written as 0 , and in general, operators of the form $\alpha \cdot 1$ where $\alpha$ is a scalar will be denoted merely by $\alpha$.

Definition 1. Take $f_{n} \in F, n=1,2, \cdots$. Then $f_{n} \rightarrow f(F)$ as $n \rightarrow \infty$ if and only if there is a $b \neq 0$ in $C^{*}$ such that $b f_{n} \in C^{*}$ for $n=1,2, \cdots$ and $b f_{n} \rightarrow b f\left(C^{*}\right)$ as $n \rightarrow \infty$.

The $F$ limit, when it exists, is unique.

$f(\lambda)$ is said to be an operator function if $f(\lambda)$ is a function whose range is in $F$. If $I^{\prime} \subset(-\infty, \infty)$ then $C\left(I^{\prime}\right) C^{*}$ is the vector space of operator functions $f(\lambda), \lambda \in I^{\prime}$, such that $f(\lambda)=\{f(\lambda, t)\}$ is continuous on $I^{\prime} \times I$. If $S$ is a region in the complex plane then $C(S) C^{*}$ is the vector space of operator functions $f(z)=\{f(z, t)\}$ which are continuous on $S \times I$. Convergence in $C\left(I^{\prime}\right) C^{*}$ and $C(S) C^{*}$ means uniform convergence on compact subsets. There are corresponding spaces $C\left(I^{\prime}\right) F$ and $C(S) F$ of operator functions. If $f(\lambda)$ (or $f(z)$ ) is such that there is a nonzero $a$ in $C^{*}$ and $a f(\lambda) \in C\left(I^{\prime}\right) C^{*}\left(C(S) C^{*}\right)$ then $f(\lambda)(f(z))$ is said to be in $C\left(I^{\prime}\right) F(C(S) F)$. A sequence $f_{n}(\lambda)$ in $C(I) F$ converges to $f(\lambda)$ as $n \rightarrow \infty$ if there is a nonzero $a$ in $C^{*}$ such that $a f_{n}(\lambda) \in C\left(I^{\prime}\right) C^{*}$ for each $n$ and this sequence converges in $C(I) C^{*}$ to $a f(\lambda)$ as $n \rightarrow \infty$. Convergence of a sequence $f_{n}(z)$ in $C(S) F$ is defined in an analogous manner.

If $f(\lambda)$ is in $C\left(I^{\prime}\right) F$ and there is a nonzero $a$ in $C^{*}$ such that $a f(\lambda)$ is not only in $C\left(I^{\prime}\right) C^{*}$ but the $(\partial / \partial \lambda)\{a f(\lambda, t)\}$ exists and is continuous on $I^{\prime} \times I$ then $f(\lambda)$ is said to be in $C_{1}\left(I^{\prime}\right) F$. If there is a nonzero $a \in C^{*}$ and $a f(z)$ is not only in $C(S) C^{*}$ but for each $t \geqq 0$, af $(z, t)$ is analytic and $(\partial / \partial z)\{a f(z, t)\}$ is in $C(S) C^{*}$ we shall say that $f(z)$ is in $A(S) F$.

Definition 2. Let $f(\lambda)$ be in $C_{1}\left(I^{\prime}\right) F$ and suppose that $a \neq 0$ is an element of $C^{*}$ such that af $(\lambda) \in C\left(I^{\prime}\right) C^{*}$. We define

$$
f^{\prime}(\lambda)=\frac{1}{a} \frac{\partial}{\partial \lambda}\{a f(\lambda, t)\} .
$$

The derivative $f^{\prime}(z)$ of an element of $A(S) F$ is defined in analogy to Definition 2.

Definition 3. Let $I^{\prime}$ be a bounded subinterval of $E^{1}$. Suppose the operator function $f(\lambda)$ to be in $C\left(I^{\prime}\right) F$. Let the scalar function $\phi(\lambda)$ be integrable over $I^{\prime}$. If $a \neq 0$ is such that $a f(\lambda)$ is in $C\left(I^{\prime}\right) C^{*}$ the integral of $\phi(\lambda) f(\lambda)$ is defined by

$$
\int_{I^{\prime}} \phi(\lambda) f(\lambda) d \lambda=\frac{1}{a}\left\{\int_{I^{\prime}} \phi(\lambda) a f(\lambda, t) d \lambda\right\} .
$$

If $f(z) \in C(S) F$ where $S$ is an open region in the complex plane and $J$ is a rectifiable curve contained in $S$, the line integral over $J$ is defined by 


$$
\int_{J} f(z) d z=\frac{1}{a}\left\{\int_{J} a f(z, t) d z\right\}
$$

where $a \neq 0$ is such that $a f(z) \in C(S) C^{*}$.

Let the scalar function $\phi(\lambda)$ be locally integrable and let the operator function $f(\lambda)$ be in $C(0 \leqq \lambda<\infty) F$. The integral of $f(\lambda) \phi(\lambda)$ on the infinite interval $[0, \infty)$ is defined by

$$
\int_{0}^{\infty} f(\lambda) \phi(\lambda) d \lambda=\lim _{T \rightarrow \infty} \int_{0}^{T} f(\lambda) \phi(\lambda) d \lambda
$$

when the limit on the right exists. The limit on the right hand side is taken in the sense of Definition 1.

The values of the integrals defined above and the derivative defined in Definition 2 do not depend on which particular element $a$ is chosen to make $a f(\lambda)$ a continuous function.

Definition 4. Suppose $\alpha<0<\beta, f(\lambda) \in C_{1}[\alpha, \beta] F, f(0)=1, w \in F$, and $(d / d \lambda) f(\lambda)=w f(\lambda)$ for all $\lambda \in(\alpha, \beta)$. In such a case $w$ is said to be a logarithm on $[\alpha, \beta]$ and $f(\lambda)=e^{\lambda w}$ on $[\alpha, \beta]$.

It is known $[9$, p. 68] that an operator $w$ which is a logarithm on an interval $\alpha \leqq \lambda \leqq \beta$ is in fact a logarithm on each finite interval $\left[\alpha_{1}, \beta_{1}\right]$ where $\alpha_{1}<\beta$, $\alpha<\beta_{1}$, and the extension for $f(\lambda)$ from $[\alpha, \beta]$ to $(-\infty, \infty)$ is unique.

Every function in $L^{*}$ is a logarithm. An example of a logarithm which is not a function is the operator $s=1 / h$. The operator $e^{-\lambda s}$ is a shift operator when $\lambda>0$. If $g=e^{-\lambda s} f, \lambda>0$, then $g(t)=0 t<\lambda, g(t)=f(t-\lambda)$ when $t \geqq \lambda$.

The operators of Mikusiński are closely related to the Laplace transform. The relationship between the Laplace transform and that subspace of $F$ which consists of all operators of the form $s^{n} a$, where $a \in C^{*}, a(t)=0\left(e^{k t}\right)$ as $t \rightarrow \infty$ and $n$ and $k$ are positive numbers, has been investigated by J. D. Weston (see [10] and [11]). The following theorem of Mikusiński suggests the existence of some relationship.

THEOREM 1. Let $f$ be locally integrable, then,

$$
\int_{0}^{\infty} e^{-\lambda s} f(\lambda) d \lambda=f .
$$

Proof. J. Mikusiński [8, pp. 337 and 377]. This theorem will be extended later to the case in which the integral exists only as a "finite part."

2.2. If an operator $w$ can be expressed as the quotient of two real, continuous functions it is said to be a real operator. If $w=e / f$ is a real operator where $e, f \in C^{*}$ then $e$ and $f$ have a common factor $c \in F$ such that $a=e / c$ and $b=f / c$ are real elements of $C^{*}$. Thus any expression of a real operator in terms of elements of $C^{*}$ is, except for common factors in the numerator and denominator, in terms 
of real functions. Every element $w \in F$ has a unique decomposition $w_{1}+w_{2} i$ where $w_{1}$ and $w_{2}$ are real. The real operators form a subfield of $F$.

The following Lemma is due to Mikusiński [8, p. 192].

LEMMA 1. Let $F(\lambda)=e^{\lambda a}$ and $F(1)=1$ then $a=2 \pi k i$ where $k$ is an integer.

Although some operators are called logarithms they are not said to be the logarithm of something. However, the preceding Lemma allows us to make the following definition.

Definition 5. Let $w$ be a real logarithm and suppose $e^{w}=a$, then $w$ is called the logarithm of $a$ (i.e., $\ln a=w$ ).

In this case the operator function $e^{\lambda w}$ is alternatively denoted by $a^{\lambda}$. It is seen by Lemma 1 that the logarithm of an operator, if it exists, is uniquely defined. If $\alpha$ is a positive scalar the operator $\ln (\alpha \cdot 1)=(\ln \alpha) \cdot 1$ where 1 is the unit element of $F$ and $\ln \alpha$ is the principal value of the logarithm of $\alpha \cdot \ln (\alpha \cdot 1)$ will be written as just $\ln \alpha$. The logarithm obeys most of the rules expected of a function with this name. The following statements are easily proved by referring to Definition 5 .

LEMMA 2.

(i) $\ln a+\ln b=\ln a b$,

(ii) $\alpha>0 ; \ln a+\ln \alpha=\ln \alpha a$,

(iii) $\alpha$ real; $\ln a^{\alpha}=\alpha \ln a$,

(iv) $\ln 1 / a=-\ln a$.

Statements (i) and (ii) are true in the sense that if any two of the quantities involved exist the third quantity exists and is given by the formula shown. Statements (iii) and (iv) are true in the sense that if one side of the equation exists the other does and is given by the formula shown. Thus the function given by $f(a)=\ln a$ is an isomorphism between the multiplicative group of operators which have logarithms and the additive group of real logarithms.

If $\lambda$ is real then $h^{\lambda}=e^{\lambda(s\{\ln t\}+C)}$, [12], where $C=.577 \cdots$ is Euler's constant. Thus, $\ln h=s\{\ln t\}+C=s\{\ln \gamma t\}$ where $\gamma=e C$. By Lemma 2 we also have $\ln s=-s\{\ln \gamma t\}$ and $\{\ln t\}=-(\ln \gamma s) / s$. Of course many operators have logarithms. The following theorem yields a large class of functions which have logarithms.

LEMMA 3. On each compact subinterval of $\lambda>-1$ the quantities $C_{\lambda, n}$ and $(\partial / \partial \lambda) C_{\lambda, n}$ converge uniformly to zero as $n \rightarrow \infty$.

Proof.

$$
C_{\lambda, n}=\frac{\Gamma(\lambda+1)}{\Gamma(n+1) \Gamma(\lambda-n+1)} \frac{\sin (n-\lambda) \Gamma(\lambda+1) \Gamma(n-\lambda)}{\Gamma(n+1)} .
$$

Stirling's formula shows $C_{\lambda, n} \rightarrow 0$ uniformly on each compact subset of $\lambda>-1$. 
To see that $(\partial / \partial \lambda) C_{\lambda, n}$ tends to zero uniformly differentiate the right hand side of the above equation and use the fact that $\Gamma^{\prime}(n-\lambda) / \Gamma(n-\lambda)=\ln |n-\lambda|$ $+O(1 / n)$.

LEMMA 4. If $a \in L^{*}$ and $\alpha_{n}, n=1,2, \cdots$, is a uniformly bounded sequence of complex numbers, then $\sum_{n=1}^{\infty} \alpha_{n} a^{n}$ is convergent $\left(L^{*}\right)$. In particular $h \sum_{n=1}^{\infty} \alpha_{n} a^{n}$ is convergent $C^{*}$.

Proof. The first statement is proved in [13]. The second statement follows immediately from the first statement.

LEMMA 5. If $\lambda>-1$ then

$$
\frac{\partial}{\partial \lambda} C_{\lambda, n}=\sum_{m=1}^{n} \frac{(-1)^{m+1}}{m} C_{\lambda, n-m} \text { for } n=1,2, \cdots
$$

Proof. This is clearly true for $n=1$. The general case follows by induction.

THEOREM 2. If $a \in L^{*}$ is real then

$$
w=\sum_{n=1}^{\infty} \frac{(-1)^{n+1}}{n} a^{n}
$$

is a logarithm and $\ln (1+a)=w$.

Proof. For $\lambda>-1$ let $f(\lambda)=\sum_{n=0}^{\infty} C_{\lambda_{n}} a^{n}$. Then $f(0)=1$. Let $g(\lambda)=h a f(\lambda)$. By Lemmas 3 and 4 the series $g(\lambda)=h \sum_{n=0}^{\infty} C_{\lambda, n} a^{n+1}$ can be differentiated term by term and the result converges in $C(\lambda>-1) C^{*}$ to $g^{\prime}(\lambda)$. Thus $f(\lambda) \in C_{1}(\lambda>-1) F$; moreover, by Lemmas 3 and 4 term by term multiplication of the series for $w$ and the series for $g(\lambda)$ is justified. Rearranging the terms and using Lemma 5 it is seen that $w g(\lambda)=w h a f(\lambda)=g^{\prime}(\lambda)=h a f^{\prime}(\lambda)$ and thus $w f(\lambda)=f^{\prime}(\lambda)$ so that $w$ is a logarithm and $f(\lambda)=e^{\lambda w}$. Since $f(1)=1+a$ we have $\ln (1+a)=w$.

COROLlary 1. If $f$ is locally absolutely continuous and $f(0)=\alpha>0$ then $\ln f$ exists.

Proof. In fact, $f=\alpha h+\left\{\int_{0}^{t} f^{\prime}(u) d u\right\}=\alpha h+f^{\prime} h=\alpha h\left(1+\left(f^{\prime} / \alpha\right)\right)$ so that $\ln f$ $=\ln \left(1+\left(f^{\prime} / \alpha\right)\right)+\ln h+\ln \alpha$.

It is not necessary that $f(t)$ be positive for every or almost every $t \geqq 0$ in order for $f$ to possess a (real) logarithm. For example

$$
\ln \{\cos t\}=\ln h+\ln (1-\{\sin t\})=s\{\ln \gamma t\}+\sum_{n=1}^{\infty} \frac{\{\sin t\}^{n}}{n} .
$$

2.3. We now introduce analytic operator functions.

Definition 6. Let $S$ be an open region in the complex plane. $f(z)$ is said to be an analytic operator function on $S$ if $f(z)$ is in $A(z) F$. 
Thus $f(z)=h^{z}$ is an analytic operator function on each half-plane $\operatorname{Re} z>\alpha$, $\alpha$ real. The operator function $\Gamma(z) h^{z}$ is analytic on each such half-plane if the nonnegative integers are deleted.

Analytic operator functions have many of the properties of analytic functions; thus

THEOREM 3. Let $f(z)$ be an analytic operator function on $S$. Then

(i) if $f(z)=0$ on a set which has an accumulation point in $S, f(z)=0$ everywhere on $S$;

(ii) if $J$ is a simple, closed, rectifiable curve in $S$ and $z$ is in the bounded region enclosed by $J$ then

$$
f^{(n)}(z)=\frac{n !}{2 \pi i} \int_{J} \frac{f(\zeta)}{(\zeta-z)^{n+1}} d \zeta
$$

(iii) if $K\left(z_{0}, p\right)=\left\{z|| z-z_{0} \mid<p\right\} \subset S$ then for $z \in K\left(z_{0}, p\right)$ we have

$$
f(z)=\sum_{n=0}^{\infty} \frac{f^{(n)}\left(z_{0}\right)}{n !}\left(z-z_{0}\right)^{n}
$$

The series is convergent $(F)$ to $f(z)$.

Proof. The proofs follow directly from the analogous theorems in complex variables. For example, to prove (ii) it is noted that for $a \neq 0$ and $a f(z)=\{g(z, t)\}$ in $A(S) C^{*}$ we have for each $t \in I$

$$
\frac{\partial^{n} g}{\partial z^{n}}(z, t)=\frac{n !}{2 \pi i} \int_{J} \frac{g(\zeta, t)}{(\zeta-z)^{n+1}} d \zeta
$$

Since $z$ is not on $J$ and $\{g(z, t)\}$ is continuous on $S \times I$, equation (1) shows that $\left(\partial^{n} / \partial z^{n}\right) g(z, t)$ is continuous at each point of $S \times I$, that is, $g^{(n)}(z)$ is in $C(S) C^{*}$. Thus

$$
f^{(n)}(z)=\frac{1}{a}\left\{\frac{\partial^{n}}{\partial z^{n}}(a f(z, t))\right\}=\frac{n !}{a}\left\{\frac{1}{2 \pi i} \int_{J} \frac{a f(\zeta, t)}{(\zeta-z)^{n+1}} d \zeta\right\}
$$

and by definition this is

$$
\frac{n !}{2 \pi i} \int_{J} \frac{f(\zeta)}{(\zeta-z)^{n+1}} d \zeta
$$

It is seen from equation (2) that an analytic operator function has the property that if $a f(z)$ is in $A(S) C^{*}$ then the same function $a$ is such that $a f^{(n)}(z)$ is in $A(S) C^{*}$ for all positive integers $n$.

In fact Theorem 3 (iii), characterizes analytic operator functions. That is, if $f_{n} \in F, n=1,2, \cdots$, are such that if $\sum_{1}^{\infty}\left(f_{n} / n !\right)\left(z_{1}-z_{0}\right)^{n}$ is convergent $(F)$ then the operator function $f(z)=\Sigma_{1}^{\infty}\left(f_{n} / n !\right)\left(z-z_{0}\right)^{n}$ is analytic on $\left|z-z_{0}\right|<\left|z_{1}-z_{0}\right|$. 
If $f(z)$ is analytic in a region which includes the origin and $f(\lambda)=e^{\lambda w}$ when $z=\lambda$ is real the power series expansion,

$$
e^{\lambda w}=\sum_{n=0}^{\infty} \frac{w^{n}}{n !} \lambda^{n}
$$

converges for sufficiently small $\lambda$. It is not true that every exponential $e^{\lambda w}$ is the restriction to the real axis of an analytic operator function.

EXAMPLE. For

$$
a \in C^{*}, \quad a e^{-\lambda s}=\left\{\begin{array}{cl}
0 & \text { if } \lambda>t \\
a(t-\lambda) & \text { if } \lambda \leqq t .
\end{array}\right.
$$

If $f(\lambda)=\{f(\lambda, t)\}$ is the restriction of an analytic operator function then $f\left(\lambda, t_{0}\right)$ must be the restriction of an analytic function. In order for

$$
f\left(\lambda, t_{0}\right)=\left\{\begin{array}{cc}
0, & \lambda>t_{0} \\
a\left(t_{0}-\lambda\right), & \lambda \leqq t_{0}
\end{array}\right.
$$

to be the restriction to the real axis of an analytic function it is necessary that $a\left(t_{0}-\lambda\right)=0$ for all $\lambda \leqq t_{0}$. Since this must be true for all $t_{0} \geqq 0$ we have $a=0$. Thus there is no nonzero $a \in C^{*}$ for which $a e^{-\lambda s} \in A(S) C^{*}$ and thus $e^{-\lambda s}$ is not in $A(S) F$ for any region $S$ which intersects the axis of reals.

THEOREM 4. Suppose that $w$ is a real operator and that both $w$ and $i w$ are logarithms. Then $e^{z w}=e^{(\lambda+i \mu) w}$ is analytic in every bounded region of the complex plane.

Proof. First we show that $e^{\lambda w}$ is real for real $\lambda$. Let $f_{1}(\lambda)$ and $f_{2}(\lambda)$ be the real and imaginary parts of $e^{\lambda w}$. Take $a \in C^{*}$ to be a nonzero real function such that $a e^{\lambda w}=a f_{1}(\lambda)+i a f_{2}(\lambda) \in C_{1}(-N \leqq \lambda \leqq N) C^{*}$ for some positive $N$. Then $a f_{1}^{\prime}(\lambda)$ $+i a f_{2}^{\prime}(\lambda)=w a e^{\lambda w}=a w f_{1}(\lambda)+i a w f_{2}(\lambda)$ on $(-N, N)$ so that both $a f_{1}^{\prime}(\lambda)=a w f_{1}(\lambda)$ and $a f_{2}^{\prime}(\lambda)=a w f_{2}(\lambda)$ on $(-N, N)$. Thus $f_{1}(\lambda)=f_{1}(0) e^{\lambda w}$ and $f_{2}(\lambda)=f_{2}(0) e^{\lambda w}$. Since $f_{1}(0)=f(0)=1$ and $f_{2}(0)=0$ it follows that $e^{\lambda w}$ is real for all real $\lambda$.

If $e^{i \mu w}=g_{1}(\mu)=i g_{2}(\mu)$ where $g_{1}(\mu)$ and $g_{2}(\mu)$ are real it can be seen in a similar manner that $g_{1}^{\prime}(\mu)=-w g_{2}(\mu)$ and $g_{2}^{\prime}(\mu)=w g_{1}(\mu)$. Moreover, let $a$ be a nonzero real function such that both $a e^{\lambda w}$ and $a e^{i \mu w}$ are in $C_{1}(-N, N) C^{*}$ for $N>0$. By use of the above two differential equations it is seen that $a e^{z w}$ satisfies the Cauchy-Riemann equations in the rectangle $-N<\operatorname{Re} z<N,-N<\operatorname{Im} z<N$ for each $t \geqq 0$. Thus, by definition, $e^{z w}$ is an analytic operator function in this region, and since such an $a$ can be found for every positive $N, e^{z w}$ is an analytic operator function in every bounded region of the $z$ plane.

Theorem 4 together with the last example confirms the well-known fact that the operator $i s$ is not a logarithm.

3.1. Theorem 3 shows the possibility of analytic continuation of analytic operator functions. 
Definition 7. Let $S_{1}$ and $S_{2}$ be nonempty regions in the complex plane and let $S_{2} \supset S_{1}$. If $f_{1}(z)$ is an analytic operator function on $S_{1}$ and $f_{2}(z)$ is an analytic operator function on $S_{2}$ such that

$$
f_{1}(z)=f_{2}(z)
$$

whenever $z \in S_{1}, f_{2}(z)$ is said to be the analytic continuation of $f_{1}(z)$ to $S_{2}$.

We will use analytic continuation in order to define the finite part of some operator functions.

Definition 8. Let $S_{1}$ and $S_{2}$ be nonempty regions in the complex plane such that $S_{2} \supset S_{1}$. Suppose that $g(z) \in A\left(S_{1}\right) C^{*}$ and $\{g(z, t)\}$ is continuous on $S_{2} \times(0, \infty)$; moreover, for $t>0, g(z, t)$ is analytic on $S_{2}$. If $f(z) \in A\left(S_{2}\right) F$ is an analytic extension of $g(z)$ as an operator function to all of $S_{2}$ then $f(z)$ is said to be the finite part of $\{g(z, t)\}$ arising from $S_{1}$. This is written

$$
[\mathrm{FP} g(z, t)]=f(z) \text {. }
$$

It is clear from the uniqueness of analytic continuation that the finite part of $\{g(z, t)\}$ arising from a given region is unique.

An example of a function whose finite part can be defined is $\{g(z, t)\}=\left\{t^{z} / \Gamma(z+1)\right\}$ where $S_{1}=\{z \mid \operatorname{Re} z>0\}$. For each positive $t$ the function $\{f(z, t)\}=\left\{t^{z} / \Gamma(z+1)\right\}$ is analytic in the entire $z$ plane and the operator function $f(z)=h^{z+1}$ is equal on $S_{1}$ to $\{g(z, t)\}$. Since $h^{z}$ is an analytic operator function in every half-plane $\operatorname{Re} z>-n$, Definition 8 gives

$$
\left[\mathrm{FP} \frac{t^{2}}{\Gamma(z+1)}\right]=h^{z+1}
$$

in any half-plane $\operatorname{Re} z>-n$.

It should be noted that a particular nonintegrable function $\{k(t)\}$, for example $\{k(t)\}=\left\{t^{-3 / 2} / \Gamma(-1 / 2)\right\}$, does not define a unique operator [FP $\left.k(t)\right]$ having the property that $[\operatorname{FP} f(z, t)]_{z=z_{1}}=[\operatorname{FP} k(t)]$ whenever $f\left(z_{1}, t\right)=k(t)$ for all positive $t$. For example

and

$$
\left[\mathrm{FP} \frac{t^{z}}{\Gamma(z+1)}+\frac{t^{z}+1 / 2}{\Gamma(z+3 / 2)}\right]_{z=3 / 2}=h^{-1 / 2}+1
$$

however

$$
\left[\mathrm{FP} \frac{t^{2}}{\Gamma(z+1)}\right]_{z=3 / 2}=h^{-1 / 2}
$$

$$
\left\{\frac{t^{z}}{\Gamma(z+1)}+\frac{t^{z}+1 / 2}{\Gamma(z+1 / 2)}\right\}_{z=-3 / 2}=\left\{\frac{t^{-3 / 2}}{\Gamma(-1 / 2)}\right\}=\left\{\frac{t^{z}}{\Gamma(z+1)}\right\}_{z=-3 / 2} .
$$

Thus when speaking of the finite part of a particular function care must be used to show how it was calculated. In spite of this the notation $[\operatorname{FP} f(\alpha, t)]$ will fre- 
quently be used in place of the more cumbersome $[\operatorname{FP} f(z, t)]_{z=\alpha}$ when there is no possibility of confusion. In particular $\left[\mathrm{FPt}^{2}\right]$ and $\left[\mathrm{FPt} t^{z} / \Gamma(z+1)\right]$ will be those analytic operator functions arrived at by using the right half-plane for $S_{1}$.

Definition 9. Let $n$ be a positive integer and suppose that $0 \leqq \beta<1$.

(i) Let $\alpha=-n-\beta$ and let the integrable function $f$ be $n$ times differentiable at the point $t$. The quantities $I_{\alpha}(f, t)$ and FP $\int_{0}^{t} f(t-u) u^{\alpha} d u$ are defined by the equation

$$
I_{\alpha}(f, t)=\mathrm{FP} \int_{0}^{t} f(t-u) u^{\alpha} d u=\lim _{\varepsilon \rightarrow 0}\left(\int_{\varepsilon}^{t} f(t-u) u^{\alpha} d u+Q(\varepsilon)\right)
$$

where $Q(\varepsilon)$ is that unique linear combination of $\ln \varepsilon$ and negative powers of: which causes the limit on the right hand side to exist.

(ii) Let the function $m$ possess $n$ derivatives each continuous on $[0, t]$. Then for some continuous function $g$

$$
m(u)=u^{n} g(u)+\sum_{k=0}^{n-1} \frac{m^{(k)}(0)}{k !} u^{n-k}
$$

on $[0, t]$. The quantity FP $\int_{0}^{t} f(t-u)\left(m(u) / u_{n+\beta}\right) d u$ is defined by the equation

$$
\mathrm{FP} \int^{t} f(t-u) \frac{m(u)}{u^{n+\beta}} d u=\int_{0}^{t} f(t-u) \frac{g(u)}{u^{\alpha}} d u+\sum_{k=0}^{n-1} \frac{m^{(k)}(0)}{k !} \mathrm{FP} \int_{0}^{t} \frac{f(t-u) d u}{u^{n-k+\beta}}
$$

whenever $f$ is such that the integrals on the right exist.

Definition 8 does not yield a value for the operator function [FPt $t^{2}$ when $z=-n$. In analogy to what has been done by Hadamard the value of the operator $\left[\mathrm{FPt}^{2}\right]$ at $z=-n$ will be defined by means of residues. The operator function [FPt ${ }^{2}$ ] has a simple pole at $z=-n$ and the residue at $z=-n$ is given by

$$
\lim _{z \rightarrow-n}(z+n)\left[\mathrm{FPt}^{2}\right]=\operatorname{Res}_{z=-n}\left[\mathrm{FP} t^{2}\right]
$$

where the limit is taken in the sense of convergence in $F$. It is seen that

$$
\operatorname{Res}_{z=-n}\left[\mathrm{FPt}^{z}\right]=\frac{(-1)^{n-1} h^{-n}}{\Gamma(n)} \text {. }
$$

The operators $\left[\mathrm{FPt}^{2}\right]_{z=-n}$ will now be defined by the equation

$$
\left[\mathrm{FPt}^{z}\right]_{z=-n}=\lim _{z \rightarrow-n}\left(\left[\mathrm{FPt} t^{z}-\frac{\operatorname{Res}_{z=-n}\left[\mathrm{FP} t^{z}\right]}{z+n}\right) .\right.
$$

Evaluating this limit gives

$$
\mathrm{FP}^{-n}=\frac{(-1)^{n-1}}{(n)} s^{n}\left\{\ln t+\gamma_{n-1}\right\}, \quad m=1,2, \cdots
$$

where $\gamma_{0}=0$ and $\gamma_{n-1}=1+1 / 2+\cdots+1 /(n-1)$ when $n>1$. The operator function [FPt ${ }^{2}$ ] as defined by Definition 8 and Equation 4 is the same as that arrived at by Butzer although he utilized quite different considerations. 
The following Lemma is well known. See for instance [4].

Lemma 6. Suppose $\beta=-n-\beta, n$ is a positive integer, $0 \leqq \beta<1, t>0$, and $f$ possesses $n$ derivatives at the point t. Then

(i) if $\beta \neq 0$

$$
\text { FP } \int_{0}^{t} f(t-u) u^{\alpha} d u=\frac{\Gamma(\alpha+1)}{\Gamma(1-\beta)} \frac{d^{n}}{d t^{n}} \int_{0}^{t} f(t-u) u^{-\beta} d u,
$$

(ii) if $\beta=0$

$$
\text { FP } \int_{0}^{t} f(t-u) u^{-n} d u=\frac{(-1)^{n-1}}{\Gamma(n)} \frac{d^{n}}{d t^{n}} \int_{0}^{t} f(t-u)\left(\gamma_{n-1}+\ln u\right) d u .
$$

In certain cases an operator which is not a function can be said to be equal to a function on a particular subinterval of $[0, \infty]$. For the following definition see Erdélyi [9, Appendix], or Mikusiński [8, Part VI, Chapter III].

Definition 10. Let there be an $n$ such that $h^{n} f \in C^{*}$. Suppose that $g=h^{n} f$ is $n$ times differentiable on the open interval $(a, b)$. Then the operator $f$ is said to be equal on $(a, b)$ to $g^{(n)}$. This is written

$$
f(t)=g^{(n)}(t) \quad \text { on }(a, b) .
$$

Thus $\left[\mathrm{FPt}^{z}\right]=t^{z}$ on $(0, \infty)$ in the above sense and if $m$ has a sufficient number of derivatives on $I$ then $\left[\mathrm{FP} m(t) / t^{2}\right]=m(t) / t^{z}$ on $(0, \infty)$. If $f$ is differentiable a sufficient number of times so that FP $\int_{0} f(t-u) u^{\alpha} d u$ exists for each $t$ on $(0, \infty)$ then this is equal in the sense of the above definition to $\left[\operatorname{FP}^{\alpha}\right] f(t)$ on $(0, \infty)$. This is an exercise in $[9$, p. 133] when $\alpha \neq-n$, but it follows for any $\alpha$ from the above definition and the preceding lemma. That this can be extended to $\left[\mathrm{FP} m(t) / t^{2}\right]$ is clear from the definitions. Thus

THEOREM 5. Let $\alpha=n+\beta$ where $n$ is a positive integer and $0 \leqq \beta<1$. Let $m$ have $n$ derivatives each of which is in $C^{*}$. Suppose $m(0) \neq 0$. Then if the locally integrable function $f$ is $n$ times differentiable on $(0, \infty)$

$$
\mathrm{FP} \int_{0}^{t} f(t-u) \frac{m(u)}{u^{\alpha}} d u=k^{(n)}(t) \quad t>0
$$

where $k$ is a locally integrable function. The operator

$$
f\left[\mathrm{FP} \frac{m(t)}{t^{\alpha}}\right]=g
$$

is such that

$$
g(t)=k^{(n)}(t) \quad \text { on }(0, \infty)
$$

in the sense of Definition 10. 
That the finite part $[\mathrm{FP} f(z, t)]$ arising from $S_{1}$ is unique is clear; however, that the operator function in $A\left(S_{1}\right) C^{*}$ which gives rise to the finite part must be completely stated including its domain of definition $S_{1}$ is seen by the following fact. We can have functions $f_{1}(z)$ and $f_{2}(z)$ which are in $A\left(S_{1}\right) C^{*}$ and $A\left(S_{2}\right) C^{*}$ respectively, where $S_{1}$ and $S_{2}$ are disjoint regions both contained in a region $S_{3}$, and such that $f_{1}(z)$ and $f_{2}(z)$ have different analytic continuations to all of $S_{3}$ as operator functions; however, for each $t>0 f_{1}(z, t)$ and $f_{2}(z, t)$ have the same analytic continuation to all of $S_{3}$. In this case it is clear that statement of the domain is necessary. An example is

$$
\begin{array}{ll}
f_{1}(z)=\frac{1}{\sqrt{ }\left(\pi t^{3}\right)} e^{-z^{2} / 4 t^{2}} & |\arg z|<\pi / 4 \\
f_{2}(z)=\frac{1}{\sqrt{ }\left(\pi t^{3}\right)} e^{-z^{2} / 4 t^{2}} & |\arg z|>3 \pi / 4
\end{array}
$$

Clearly, for each $t>0$ these functions have the same analytic continuation to the entire complex plane. In [8, Chapter VIII] it is shown that both $\sqrt{ } s$ and $i \sqrt{ } s$ are logarithms and by Theorem 4 we know that $e^{-z \sqrt{ } s}=e^{-x \sqrt{ } s} e^{-i y \sqrt{ } s}$ is an analytic operator function in every bounded region of the complex plane; thus, $e^{-z \sqrt{ } s} / z$ is an analytic operator function in every bounded region of the complex plane which does not include the origin. It is known [8, pp. 221-222] that

$$
\frac{e^{-z \sqrt{ } s}}{z}=\frac{e^{-x \sqrt{ } s}}{x}=\left\{\frac{1}{\sqrt{ }\left(\pi t^{3}\right)} e^{-x^{2} / 4 t^{2}}\right\}
$$

when $z=x>0$. Since $f_{1}(z)$ is equal to $e^{-z \sqrt{ } s} / z$ for an infinite number of $z$ (all positive $z$ ) we know by Theorem 3 (i), that $f_{1}(z)=e^{-z \sqrt{ } s} / z$ in the entire region $|\arg z|<\pi / 4$. Since this is an analytic operator function in any bounded region not including the origin we have that the finite part of $\left\{e^{-z^{2} / 4 t^{2}} / \sqrt{ }\left(\pi t^{3}\right)\right\}$ arising from $|\arg z|<\pi / 4$ is

$$
\left[\mathrm{FP} \frac{e^{-z^{2} / 4 t^{2}}}{\sqrt{\left(\pi t^{3}\right)}}\right]=\frac{e^{-z \sqrt{ } s}}{z} .
$$

On the other hand the finite part arising from $|\arg z|>3 \pi / 4$ is

$$
\left[\operatorname{FP} \frac{e^{-z^{2} / 4 t^{2}}}{\sqrt{ }\left(\pi t^{3}\right)}\right]=-\frac{e^{z \sqrt{ } s}}{z} .
$$

If $[\operatorname{FP} f(z, t)]$ is of a particular type, namely if $[\operatorname{FP} f(z, t)]=s^{n} g(z)$ on $S_{2}$ where $g(z) \in A\left(S_{2}\right) C^{*}$, it is not necessary to specify the domain $S_{1}$ from which the finite part arises. This follows from

THEOREM 6. Let $S_{1}$ and $S_{2}$ be regions in the complex plane, $S_{2} \supset S_{1}$. If $f(z) \in A(S) C^{*}, g(z)=A\left(S_{2}\right) C^{*}$ and suppose that the finite part of $\{f(z, t)\}$ 
arising from $S_{1}$ is $[\mathrm{FP} f(z, t)]=s^{n} g(z)$ on $S_{2}$. Then for each $t>0,\left(\partial^{n} / \partial t^{n}\right) g(z, t)$ exists and

$$
\frac{\partial^{n}}{\partial t^{n}} g(z, t)=f(z, t) \quad \text { for } z \in S_{2}
$$

Proof. Suppose that $n=1$. The case for general $n$ follows by induction. When $n=1$ we have

$$
g(z, t)=\int_{0}^{t} f(z, u) d u, \quad z \in S_{1}, t \in I .
$$

For $t>\varepsilon>0, \int_{\varepsilon}^{t} f(z, u) d u$ is analytic in $S_{2}$ and the derivative with respect to $t$ is $f(z, t)$. Now $g(z, t)$ and $\int_{\varepsilon}^{t} f(z, u) d u$ differ only by a constant then on $S_{2}$. Thus $g(z, t)$ possesses a derivative with respect to $t$ when $t>0$ and this derivative is equal to $f(z, t)$.

In particular if $[\mathrm{FP} f(z, t)]=s^{n} g(z)$ in $S_{2}$ is the finite part of $f(z, t)$ arising from $S_{1} \subset S_{2}$ then the finite part arising from any region $S_{3} \subset S_{2}$ is also $s^{n} g(z)$.

Definition 3 tells how to form the integral with respect to a parameter of an operator function. In order to get an analogue of Theorem 1 in the case of divergent integrals we need the following definition.

Definition 11. Suppose $f(\lambda) \in C_{n}[0, \mu] F$. Take a nonzero element $a$ in $C^{*}$ such that $a f(\lambda) \in C_{n}[0, \mu] C^{*}$. The integral FP $\int_{0}^{\mu}\left(f(\lambda) / \lambda^{\alpha}\right) d \lambda$ where $\alpha=n+\beta, n \geqq 1$ is an integer, and $0 \leqq \beta<1$ is defined by

$$
\operatorname{FP} \int_{0}^{\mu} \frac{f(\lambda)}{\lambda^{\alpha}} d \lambda=\frac{1}{a}\left\{\operatorname{FP} \int_{0}^{\mu} \frac{a f(\lambda, t)}{\lambda^{\alpha}} d \lambda\right\} .
$$

If the limit in the sense of convergence in $F$ exists as $\mu \rightarrow \infty$ then

$$
\mathrm{FP} \int_{0}^{\infty} \frac{f(\lambda)}{\lambda^{\alpha}} d \lambda=\lim _{\mu \rightarrow \infty} \operatorname{FP} \int_{0}^{\mu} \frac{f(\lambda)}{\lambda^{\alpha}} d \lambda .
$$

The improper integral FP $\int_{0}^{\mu}\left(a f(\lambda, t) / \lambda^{\alpha}\right) d \lambda$ exists and is in $C^{*}$ so that the definition makes sense. Moreover, the integral is independent of the function $a$ so long as $a$ has the properties required of it in the definition. The transformation $T(f(\lambda))$ $=\mathrm{FP} \int_{0}^{\mu}\left(f(\lambda) / \lambda^{\alpha}\right) d \lambda$ is a linear transformation on $C_{n}[0, \mu] F$.

TheOREM 7. Let $\alpha=n+\beta$ where $n \geqq 1$ is an integer and $0 \leqq \beta<1$. Suppose that $m^{(k)} \in C^{*}$ for $k=0,1, \cdots, n$; then

$$
\mathrm{FP} \int_{0}^{\infty} e^{-\lambda s} \frac{m(\lambda)}{\lambda^{\alpha}} d \lambda=\left[\mathrm{FP} \frac{m(t)}{t^{\alpha}}\right] .
$$

Proof. Let $\{m(t)\}=h$. Suppose $\beta=0$. Then

$$
\operatorname{FP} \int_{0}^{\mu} \frac{e^{-\lambda s}}{\lambda^{n}} d \lambda=s^{n+1} \operatorname{FP} \int_{0}^{\mu} \frac{h^{n+1} e^{-\lambda s}}{\lambda^{n}} d \lambda=\frac{s^{n+1}}{\Gamma(n+1)} I(\mu)
$$


where

Thus,

$$
\begin{array}{ll}
I(\mu, t)=\operatorname{FP} \int_{0}^{\mu} \frac{(t-\lambda)^{n}}{\lambda^{n}} d \lambda & \text { when } \mu<t, \\
I(\mu, t)=\operatorname{FP} \int_{0}^{t} \frac{(t-\lambda)^{n}}{\lambda^{n}} d \lambda & \text { when } \mu \geqq t .
\end{array}
$$

$$
I(\mu, t)=(-1)^{n-1} n t \ln t+t \sum_{k=2}^{n} \frac{(-1)^{n-k-1}}{k-1} C_{n, k}, \quad \mu \geqq t
$$

which after some computation is seen to be

$$
I(\mu, t)=(-1)^{n-1} n\left(t \ln t-t+\gamma_{n-1} t\right), \quad t \leqq \mu
$$

so that $I(\mu) \in C^{*}$ for each $\mu>0$ and $I(\mu) \rightarrow(-1)^{n-1} n h\left\{\ln t+\gamma_{n-1}\right\}(F)$

$$
\mathrm{FP} \int_{0}^{\infty} \frac{e^{-\lambda s}}{\lambda^{n}} d \lambda=\frac{(-1)^{n-1}}{\Gamma(n)} s^{n}\left\{\ln t+\gamma_{n-1}\right\}
$$

which proves the theorem in the case $m=h, \beta=0$.

When $m=h, \beta \neq 0$ the proof is similar. Since

$$
\frac{m(\lambda)}{\lambda^{\alpha}}=\frac{g_{n}(\lambda)}{\lambda^{\alpha}}+\sum_{k=0}^{n-1} \frac{m^{(k)}(0)}{k !} \lambda^{k-\alpha}
$$

where $\left\{g_{n}(t) / t^{\alpha}\right\}$ is locally integrable. Theorem 1 together with the special case of Theorem 7 just proved shows that

$$
\mathrm{FP} \int_{0}^{\infty} e^{-i s} \frac{m(\lambda)}{\lambda^{\alpha}} d \lambda=\left\{\frac{g_{n}(t)}{t^{\alpha}}\right\}+\sum_{k=0}^{n-1} \frac{m^{(k)}(0)}{k !}\left[\mathrm{FP} t^{k-\alpha}\right]
$$

and this is the statement of Theorem 7.

3.2. We give a short table of some operator functions which are finite parts.

Table Of Finite Parts Of Functions

$$
\{f(z, t)\} \quad[\operatorname{FPf}(z, t)] \quad \text { Region of }
$$

1. $\left\{\frac{t^{z-1}}{\Gamma(z)}\right\} \quad h^{z} \quad$ all $z$
2. $\left\{t^{2}\right\}$
$\Gamma(z+1) h^{z+1}$
$z \neq-1,-2, \cdots$
$\left\{t^{-n}\right\}$
$\frac{(-1)^{n-1} s^{n}}{\Gamma(n)}\left\{\ln t+\gamma_{n-1}\right\}$
$z=-n$,
$n=1,2, \cdots$ 
3. $\left\{t^{z} \ln t\right\} \quad(\psi(z+1)+s\{\ln \gamma t\})\left[\mathrm{FP}^{2}\right]$

$$
\left\{t^{-n} \ln t\right\} \quad\left(\gamma_{n}+s\{\ln t\}\right)\left[\mathrm{FP} t^{-n}\right]
$$

$z=-n$

4. $\left\{\frac{J_{0}(t)}{t^{z}}\right\}$

$$
\begin{aligned}
\left\{J_{0}(t)\right. & \left.-\sum_{n=0}^{n_{1}} \frac{(-1)^{n} t^{2 n}}{2^{2 n}(n !)^{2}}\right\} \\
& +\sum_{n=0}^{n_{1}} \frac{(-1)^{n}\left[\mathrm{FP} t^{2 n-2}\right]}{2^{2 n}(n !)^{2}}
\end{aligned}
$$

$\operatorname{Re}\left(2_{n_{1}}-z\right)>-3$

5. $\left\{\frac{e^{-z^{2} / 4 t}}{\pi^{1 / 2} t^{3 / 2}}\right\} \frac{e^{-z \downarrow s}}{z}$ arising from $|\arg z|<\pi / 4$

$z \neq 0$

$$
\begin{array}{rlrl}
\gamma_{0} & =0, & \gamma_{n-1} & =1+\frac{1}{2}+\cdots+\frac{1}{n-1} \\
\gamma & =e^{c}, & C=.577 \cdots \text { is Euler's constant. } \\
\psi(z) & =\frac{\Gamma^{\prime}(z)}{\Gamma(z)} . &
\end{array}
$$

when $n \geqq 1$.

All of the entries in the table except number 3 have already been discussed. Entry 4 is an example of the more general $\left[\mathrm{FP} m(t) / t^{2}\right]$. A direct application of Definition 9 gives entry 3 for $z$ not a negative integer and for the negative integers the entry is determined by residues.

4.1. The inverses of several of the operators which represent finite parts have been found by Butzer [7]. If $\alpha=-n-\beta, 0<\beta<1$ and $n$ is a positive integer the operator

$$
\left[\mathrm{FP} t^{\alpha}\right]^{-1}=\frac{h^{n+\beta-1}}{\Gamma(\alpha+1)}
$$

is a locally integrable function. The inverse of $\left[\mathrm{FP} t^{-1}\right]=s\{\ln t\}$ is $\int_{0}^{\infty} t^{u-1} / \gamma^{u} \Gamma(u) d u$ which is also a locally integrable function. For $n \geqq 1$

$$
\left[\mathrm{FP}^{-n}\right]^{-1}=(-1)^{n-1} \Gamma(n)\left\{\int_{0}^{\infty} \frac{t^{u-1}}{\Gamma(u) \alpha_{n-1}^{u}} d u\right\} h^{n-1}, \quad n=1,2, \cdots
$$

where $\gamma_{n-1}=\gamma e^{-\gamma n-1}$, and for $n>1$ these functions are loccally absolutely continuous. These facts enable one to find the inverse of $[\mathrm{FP} m(t) / t]$.

Lemma 7. Suppose $m^{(k)} \in C^{*}$ when $k=0,1, \cdots, n \geqq 1$ is an integer, $\alpha=n+\beta$ where $0 \leqq \beta<1$. Suppose that at least one of the quantities $m^{(k)}(0), k=0,1, \cdots, n-1$ is not zero. Then $\left[\mathrm{FP} m(t) / t^{\alpha}\right]^{-1}$ is a locally integrable function.

Proof. Let $k_{1}$ be the first integer such that $m^{\left(k_{1}\right)}(0) \neq 0$. From $\S 3.2$ we know that 


$$
\left[\mathrm{FP} \frac{m(t)}{t^{\alpha}}\right]=g_{n}+\sum_{k=k_{1}}^{n-1} \frac{m^{(k)}(0)}{k !}\left[\mathrm{FP} t^{k-\alpha}\right]
$$

where $g_{n} \in C^{*}$. Let

and

$$
f=\left(g_{n}+\sum_{k=k_{1}+1}^{n-1} \frac{m^{(k)}(0)}{k !}\left[\mathrm{FP} t^{k-\alpha}\right]\right)\left[\mathrm{FP} t^{k_{1}-\alpha}\right]^{-1}
$$

$$
\beta=\frac{m^{(k)}(0)}{k_{1} !}
$$

Then $f \in C^{*}$ and $\beta \neq 0$ is a scalar. We have

$$
\left[\mathrm{FP} \frac{m(t)}{t^{\alpha}}\right]^{-1}=\frac{\left[\mathrm{FP} t^{k_{1}-\alpha}\right]^{-1}}{f+\beta}
$$

The inverse of $f+\beta$ is

$$
\frac{1}{\beta} \sum_{n=0}^{\infty}(-1)^{n}\left(\frac{f}{\beta}\right)^{n}=\frac{1}{\beta}+\sum_{n=1}^{\infty} \frac{(-1)^{n} f^{n}}{\beta^{n+1}}
$$

Since $f \in C^{*}$ the last sum is likewise in $C^{*}$. Thus,

$$
\left[\mathrm{FP} \frac{m(t)}{t^{\alpha}}\right]^{-1}=\frac{1}{\beta}\left[\mathrm{FP} t^{k_{1}-\alpha}\right]^{-1}+\left[\mathrm{FPt}^{k_{1}-\alpha}\right]^{-1}\left(\sum_{k=1}^{\infty} \frac{(-1)^{n} f^{n}}{\beta^{n+1}}\right)
$$

is locally integrable since it is the sum of a locally integrabie function and a continuous function.

It can now be seen how to use operators in order to solve singular integral equations.

THEOREM 8. Let $\alpha=-n-\beta$, where $0 \leqq \beta<1$, and $n$ is a positive integer. A necessary and sufficient condition that there exist a function $f$ such that

$$
\mathrm{FP} \int_{0}^{t} f(t-u) u^{\alpha} d u=g(t) \quad \text { all } t>0
$$

is that both of the following conditions be satisfied:

(i) $g$ is the nth derivation on $(0, \infty)$ of a function $k$ which is locally integrable;

(ii) the operator $s^{n} k\left[\mathrm{FP} t^{\alpha}\right]^{-1}$ is a locally integrable function which is $n$ times differentiable at each point of the interval $(0, \infty)$.

If conditions (i) and (ii) are satisfied all of the solutions to Equation (7) are given by

$$
f=f_{1}+f_{0}
$$

where

$$
f_{1}(t)=\left(s^{n} k\left[\mathrm{FP}^{\alpha}\right]^{-1}\right)(t) \quad \text { on }(0, \infty)
$$

and $f_{0}$ is any function such that 


$$
f_{0}(t)=\left(\left(\alpha_{0}+\alpha_{1} s+\cdots+\alpha_{n-1} s^{n-1}\right)\left[\mathrm{FP} t^{\alpha}\right]^{-1}\right)(t) \quad \text { on }(0, \infty)
$$

where the $\alpha$ 's are complex numbers. The functions are equal to the operators in the sense of Definition 10.

Proof. First we show that the set of all solutions to the homogeneous equation

is given by Equation (9).

$$
\mathrm{FP} \int_{0}^{t} f(t-u) u^{\alpha} d u=0 \quad \text { all } t>0
$$

Let $f$ be a solution to the homogeneous equation. By Lemma 9 the operator $f\left[\mathrm{FPt}^{\alpha}\right]$ is zero on the interval $(0, \infty)$ and thus it is a polynomial in $s$. We have

$$
f=\left[\mathrm{FP} t^{\alpha}\right]^{-1}\left(\sum_{k=0}^{p} \alpha_{k} s^{k}\right)
$$

for some integer $p . f$ must be at least locally integrable in order that FP $\int_{0}^{t} f(t-u) u^{\alpha} d u$ exist according to Definition 9. If $f$ is locally integrable it must be that $p \leqq n-1$. Thus every solution to the homogeneous equation must be of the form given in Equation (9).

From Equations (5) and (6) it is seen that the operator specified in Equation (9) is indeed a locally integrable function which is infinitely differentiable for each positive $t$. Thus FP $\int_{0}^{t} f_{0}(t-u) u^{\alpha} d u$ is defined by Definition 9 for each positive $t$. The operator $f_{0}\left[\mathrm{FPt}^{\alpha}\right]=\sum_{0}^{n-1} \alpha_{i} s^{i}$ is equal to zero on $(0, \infty)$ in the sense of Definition 10. Thus by Theorem 5

$$
\text { FP } \int_{0}^{t} f_{0}(t-u) u^{\alpha} d u=0 \quad \text { all } t>0 .
$$

Thus we have proved that the set of all solutions to the homogeneous equation is given by Equation (9). Let $f$ be any solution to Equation (7). A function $\bar{f}$ is then a solution to Equation (7) if and only if $f=\bar{f}+f_{0}$ where $f_{0}$ is a solution to the homogeneous equation. Thus, if there is one solution we can find all the solutions. We will now show that conditions (i) and (ii) together are necessary and sufficient conditions in order that there exist one solution to Equation (7).

Suppose that (i) and (ii) are satisfied. Let $f_{1}=s^{n} k\left[\mathrm{FPt} t^{\alpha}\right]^{-1}$. Since $f_{1}$ is $n$ times differentiable the integral FP $\int_{0}^{t} f_{1}(t-u) u^{\alpha} d u$ exists for each $t>0$. Define $g_{1}$ by

Since

$$
\operatorname{FP} \int_{0}^{t} f_{1}(t-u) u^{\alpha} d u=g_{1}(t) \quad \text { all } t>0 \text {. }
$$

$$
f_{1}\left[\mathrm{FPt}^{\alpha}\right]=s^{n} k
$$

it is seen by Theorem 5 that $s^{n} k(t)=g_{1}(t)$ on $(0, \infty)$ in the sense of Definition 10 . But $s^{n} k(t)=g(t)$ on $(0, \infty)$ so that

$$
g(t)=g_{1}(t) \quad \text { all } t>0 .
$$


Thus $f_{1}$ is a solution to Equation (7).

On the other hand if Equation (7) has a solution, Theorem 5 shows that (i) is satisfied, that is $g(t)=k^{(n)}(t)$ for each $t>0$ for some locally integrable function $k$; this solution $f$ is such that

$$
f\left[\mathrm{FP} t^{\alpha}\right]=g_{2}
$$

for some operator $g_{2}$ and

$$
g_{2}(t)=k^{(n)}(t)
$$

on $(0, \infty)$ in the sense of Definition 10 . Thus

$$
\frac{d^{n}}{d t^{n}}\left(h^{n} g_{2}\right)(t)=k^{(n)}(t)
$$

at each point of $(0, \infty)$. Solving this differential equation we get

Thus

$$
h^{n} g_{2}=k+\sum_{r=1}^{n} \alpha_{n-r} h^{r}
$$

and

$$
g_{2}=s^{n} k+\sum_{r=1}^{n} \alpha_{n-r} h^{k-n}
$$

$$
g_{2}\left[\mathrm{FP} t^{\alpha}\right]^{-1}-s^{n} k\left[\mathrm{FP} t^{\alpha}\right]^{-1}=f-s^{n} k\left[\mathrm{FP} t^{\alpha}\right]^{-1}=\left(\sum_{0}^{n-1} \alpha_{r} s^{r}\right)\left[\mathrm{FPt}^{\alpha}\right]^{-1} .
$$

$f$ satisfies Equation (8) and the right hand side of the above equation satisfies the homogeneous equation, thus the operator $s^{n} k\left[\mathrm{FP}^{\alpha}\right]^{-1}$ satisfies condition (ii) and Equation (7). This completes the proof of the theorem.

These same methods may be used to solve singular convolution equations where the convolution involves $m(t) /\left(t^{n+\beta}\right)$ rather than $1 /\left(t^{n+\beta}\right)$. The fact that the operator $\left[\mathrm{FP} m(t) / t^{\alpha}\right]$ can be expressed in terms of the operators $\left[\mathrm{FPt} t^{k-\alpha}\right]$ by the representation

$$
\left[\mathrm{FP} \frac{m(t)}{t^{\alpha}}\right]=g_{n}+\sum_{k=0}^{n-1} \frac{m^{(k)}(0)}{k !}\left[\mathrm{FP}^{k-\alpha}\right]
$$

allows us to state a lemma analogous to Lemmas 7 and 8 but involving [FP $\left.m(t) / t^{\alpha}\right]$ rather than $\left[\mathrm{FPt}^{\alpha}\right]$.

TheOREM 9. Let $\alpha=n+\beta, n$ positive integer, $0 \leqq \beta<1$. Let $m$ be a continuous function on $I$ with $n$ continuous derivatives. Suppose $m(0) \neq 0$. A necessary and sufficient condition that there exist a solution $f$ to the equation.

$$
\mathrm{FP} \int_{0}^{t} f(t-u) \frac{m(t)}{t^{\alpha}} d u=g(t) \quad \text { all } t>0
$$

is that both of the following conditions be satisfied: 
(i) $g$ is the nth derivative on $(0, \infty)$ of a locally integrable function $k$;

(ii) the operator $s^{n} k\left[\mathrm{FP} m(t) / t^{\alpha}\right]^{-1}$ is a locally integrable function which is $n$ times differentiable on $(0, \infty)$.

Every solution is of the form

$$
f=f_{1}+f_{0}
$$

where $f_{1}(t)=\left(s^{n} k\left[\mathrm{FP} m(t) / t^{\alpha}\right]^{-1}\right)(t)$ on $(0, \infty)$ and $f_{0}$ is a solution to the homogeneous equation

$$
\mathrm{FP} \int_{0}^{t} f(t-u) \frac{m(t)}{t^{\alpha}} d u=0 \quad \text { all } t>0
$$

The proof is essentially the same as the proof of Theorem 8 and will not be given again.

By Lemma $7\left[\mathrm{FP} m(t) / t^{\alpha}\right]^{-1}$ is locally integrable and if it is $n$ times differentiable on $(0, \infty)$ there will be nonzero solutions to the homogeneous equation. The functions $f=\alpha_{0}\left[\mathrm{FP} m(t) / t^{\alpha}\right]^{-1}$ with $\alpha_{0}$ a complex number are such that

$$
f\left[\operatorname{FP} \frac{m(t)}{t^{\alpha}}\right]=\alpha_{0}
$$

and the operator $\alpha_{0}$ is equal to zero on $(0, \infty)$ in the sense of Definition 10. Thus

Theorem 5 these functions satisfy the homogeneous equation. The dimension of the vector space of solutions to the homogeneous equation is dependent on the order to which $m^{\prime}$ vanishes at the origin. As we have seen when $m=h$ (i.e., $m^{\prime}(0), \cdots, m^{(n-1)}(0)=0$ ) the dimension is $n$.

4.2 The results discussed in the preceding section will be applied in this section to solve the particular singular integral equation

$$
\mathrm{FP} \int_{0}^{t} f(t-u) \frac{J_{0}(u)}{u} d u=g(t) \quad \text { all } t>0
$$

He re $J_{0}$ is the Bessel function of the first kind and of order zero.

The operator $r=\left(s^{2}+1\right)^{1 / 2}$ is defined by the power series expansion of $\left.s^{2}+1\right)^{1 / 2}$, and from Mikusiński $[8$, p. 456] it is known that

$$
(r-s)^{2 n}=\left\{\frac{2 n}{t} J_{2 n}(t)\right\} \quad n=1,2, \cdots
$$

where $J_{2 n}$ is the Bessel function of order $2 n$. In the remainder of this section we shall use the symbol $r$ to denote $\left(s^{2}+1\right)^{1 / 2}$ and the symbol $\rho$ to denote $\left(z^{2}+1\right)^{1 / 2}$ where $z$ is a complex number. If $a$ is a locally integrable function which has a Laplace transform we shall denote its Laplace transform, $\int_{0}^{\infty} e^{-z t} a(t) d t$, by $\bar{a}(z)$.

THEOREM 10. (i) $\left[\mathrm{FP} J_{0}(t) / t\right]=\ln 2(r-s) / \gamma$.

(ii) Let a be the locally integrable function $\left[\mathrm{FP} J_{0}(t) / t\right]^{-1}$. We have 
when $\operatorname{Re} z>0$.

$$
\bar{a}(z)=\frac{1}{\ln \frac{2}{\gamma}(\rho-z)}
$$

Proof. (i) It is shown in Erdélyi [14, p. 26] that

Thus

$$
J_{0}(t)+2 \sum_{n=1}^{\infty} J_{2 n}(t)=1 \text {. }
$$

$$
\begin{aligned}
\left\{\frac{J_{0}(t)-1}{t}\right\} & =-\left\{\sum_{n=1}^{\infty} \frac{2}{t} J_{2 n}(t)\right\} \\
& =-\sum_{n=1}^{\infty} \frac{1}{n}\left\{\frac{2 n}{t} J_{2 n}(t)\right\} \\
& =-\sum_{n=1}^{\infty} \frac{(r-s)^{2 n}}{n}
\end{aligned}
$$

and the series is convergent $\left(C^{*}\right)$ since $(r-s)^{2} \in C^{*}$. Thus

$$
\left\{\frac{J_{0}(t)-1}{t}\right\}=\ln \left(1-(r-s)^{2}\right)=\ln 2 s(r-s) \text {. }
$$

Now

which proves (i).

$$
\begin{aligned}
{\left[\mathrm{FP} \frac{J_{0}(t)}{t}\right] } & =\left\{\frac{J_{0}(t)-1}{t}\right\}+\left[\mathrm{FP}^{-1}\right] \\
& =\ln 2 s(r-s)-\ln \gamma s \\
& =\ln \frac{2}{\gamma}(r-s)
\end{aligned}
$$

(ii) Let

and

$$
a=\left[\mathrm{FP} \frac{J_{0}(t)}{t}\right]^{-1}, \quad b=\left\{\frac{J_{0}(t)-1}{t}\right\}
$$

$$
c=\left[\mathrm{FP} t^{-1}\right]^{-1}=\left\{\int_{0}^{\infty} \frac{t^{u-1}}{\Gamma(u) \gamma^{u}} d u\right\} .
$$

From the proof of Lemma 7 we know that

$$
a=\frac{c}{1+c b}=c+c \sum_{n=1}^{\infty}(-1)^{n}(c b)^{n} .
$$

The function $c$ possesses a Laplace transform (Erdélyi [14, p. 251, Equation (11)]); thus if the sum on the right (which represents a continuous function) is exponentially bounded, $a$ possesses a Laplace transform. We will first show this sum to be exponentially bounded. 
Since $\left|J_{0}(t)\right| \leqq 1$ for all $t$ and $b \in C^{*}$ there is a constant $B$ such that $|b|(t) \leqq B$ for all $t \geqq 0$. Since $c(t)$ is positive for $t>0$

$$
|c b|(t) \leqq B \int_{0}^{t} c(\xi) d \xi=B \int_{0}^{t} \int^{\infty} \frac{\xi^{u-1}}{\Gamma(u) \gamma^{u}} d u d \xi .
$$

The integral $\int_{0}^{\infty}(u-1) / \Gamma(u) \gamma^{u} d u$ converges uniformly on each interval $0 \leqq \xi \leqq t$ so that

$$
\int_{0}^{t} \int_{0}^{\infty} \frac{\xi^{u-1}}{\Gamma(u) \gamma^{u}} d u d \xi=\int_{0}^{\infty} \int_{0}^{t} \frac{\xi^{u-1}}{\Gamma(u) \gamma^{u}} d \xi d u=\int_{0}^{\infty} \frac{t^{u}}{\Gamma(u+1) \gamma^{u}} d u .
$$

This is a well known function $v(t / \gamma)$, and from Erdélyi [14, p. 219]

$$
0 \leqq v\left(\frac{t}{\gamma}\right)=\int_{0}^{\infty} \frac{t^{u}}{\Gamma(u) \gamma^{u}} d u \leqq e^{t / \gamma}, \quad t>0 .
$$

Thus

$$
|c b|(t) \leqq B e^{t / \gamma}, \quad t>0
$$

and

$$
\sum_{n=1}^{\infty}|c b|^{n}(t) \leqq \sum_{n=1}^{\infty} \frac{B^{n} t^{n-1}}{\Gamma(n)} e^{t / \gamma}=B e^{((B+\gamma) / \gamma) t} .
$$

Thus the function $a$ possesses a Laplace transform.

In order to evaluate $\bar{a}(z)$ we note that $J_{0}(z)=1 / \rho$ and the Laplace transform of $\left\{J_{0}(t)-1\right\}$ is $1 / \rho-1 / z$. Since $b=\left\{\left(J_{0}(t)-1\right) / t\right\}$

$$
\bar{b}(z)=\int_{z}^{\infty} \frac{1}{\sqrt{ }\left(u^{2}+1\right)}-\frac{1}{u} d u=\ln \frac{2 z}{z+\rho} .
$$

Thus the transform of $h\left[\mathrm{FP}\left(J_{0}(t) / t\right)\right]=h b+\{\ln t\}$ is

$$
h\left[\mathrm{FP} \frac{J_{0}(t)}{t}\right](z)=\frac{1}{z} \ln \frac{2 z}{z+\rho}-\frac{\ln \gamma z}{z}=\frac{1}{z} \ln \frac{2}{\gamma}(\rho-z) .
$$

Now

$$
\bar{a}(z) h \overline{\left[\mathrm{FP} \frac{J_{0}(t)}{t}\right]}(z)=a h \overline{\left[\mathrm{FP} \frac{J_{0}(t)}{t}\right]}(z)=h(z)=\frac{1}{z}, \quad \operatorname{Re} z>0
$$

and we have

which proves (ii).

$$
\bar{a}(z)=\frac{1}{\ln (2(\rho-z) / \gamma)}, \quad \operatorname{Re} z>0
$$

A more explicit representation of $\left[\mathrm{FP} J_{0}(t) / t\right]^{-1}$ can be obtained from the inversion formula for Laplace transforms. To get convergence of the integral which occurs we will use the representation for $h^{2}\left[\mathrm{FP} J_{0}(t) / t\right]^{-1}$. Thus 
whenever $c>0$.

$$
\begin{aligned}
{\left[\mathrm{FP} \frac{J_{0}(t)}{t}\right]^{-1} } & =\left\{\frac{d^{2}}{d t^{2}}\left(h^{2}\left[\mathrm{FP} \frac{J_{0}(t)}{t}\right]^{-1}\right)\right\}(t) \\
& =\left\{\frac{d^{2}}{d t^{2}} \frac{1}{2 \pi i} \int_{c-i \infty}^{c+i \infty} \frac{e^{z t} d z}{z^{2} \ln (2(\rho-z) / \gamma)}\right\}
\end{aligned}
$$

We already know that a necessary and sufficient condition that the equation

$$
\mathrm{FP} \int_{0}^{t} f(t-u) \frac{J_{0}(y)}{u} d u=g(t) \quad \text { all } t>0
$$

possess a solution is that both the following conditions hold: (i) there is a locally integrable function $k$ which is differentiable on $(0, \infty)$ and is such that $k^{\prime}(t)=g(t)$ for all $t>0$, and (ii) $s\left[F P J_{0}(t) / t\right]^{-1} k$ is locally integrable and differentiable on $(0, \infty)$.

COROllary 2. Let the conditions (i) and (ii) hold. Let $k$ be as specified in (i), let $c>0$ and

$$
a(t)=\frac{d^{2}}{d t^{2}} \frac{1}{2 \pi i} \int_{c-i \infty}^{c+i \infty} \frac{e^{z t} d z}{z^{2} \ln (2(\rho-z) / \gamma)} \quad \text { all } t>0
$$

A necessary and sufficient condition that $f$ be a solution to Equation (10) is that for some complex number $\alpha_{0}$

$$
f(t)=(a k)^{\prime}(t)+\alpha_{0} a(t) \quad \text { all } t>0 .
$$

Proof. Since $\left[\mathrm{FP} J_{0}(t) / t\right]^{-1}=\{a(t)\}$ it follows from Theorem 9 that $f(t)$ satisfies Equation (10). It only remains to show that the solutions to the homogeneous equation form a vector space of exactly one dimension. The function

$$
a=\frac{1}{\left\{\left(J_{0}(t)-1\right) / t\right\}+\left[\overline{F P^{-1}}\right]}
$$

is the sum of a locally integrable function $\left[\mathrm{FPt}^{-1}\right]^{-1}$ and an infinite series which represents a continuous function. Since $\left[\mathrm{FPt}^{-1}\right]^{-1}=\int_{0}^{\infty}\left(\left(t^{u-1}\right) / \Gamma(u) \gamma^{u}\right) d u$ is discontinuous at the origin, $s a$ is not a function for any $n \geqq 1$, and the only solutions to the homogeneous equation are scalar multiples of $a$.

\section{REFERENCES}

1. Sur un nouveau genre d'integrales, Oeuvres complètes d'Augustin Cauchy, Series II, Vol. 6, pp. 78-88, Gauthier-Villars, Paris, 1938.

2. Diverses proprietés de la fonction $\Gamma(x)$, Oeuvres complètes d'Augustin Cauchy, Series II, Vol. 7, pp. 121-123, Gauthier-Villars, Paris, 1938.

3. J. Hadamard, Lectures on Cauchy's problem in linear hyperbolic differential equations, Dover, New York, 1953.

4. F. Bureau, Divergent integrals and partial differential equations, Comm. Pure and Appl. Math. 8 (1955), 143-202. 
5. L. Schwartz, Theorie des distributions, Vols. I and II, Hermann, Paris, 1957.

6. M. Lighthill, Introduction to Fourier analysis and generalized functions, Cambridge Univ. Press Cambridge, 1959.

7. P. Butzer, Singular integral equations of Volterra type and the finite part of divergent integrals, Arch. Rational Mech. Anal. 3 (1959), 194-205.

8. J. Mikusiński, Operational calculus, Pergamon, New York, 1959.

9. A. Erdélyi, Operational calculus and generalized function, California Institute of Technology, 1959. (Lecture notes)

10. J. D. Weston, Operational calculus and generalized functions, Proc. Roy. Soc. London Ser. A 250 (1959), 460-471.

11. — An extension of the Laplace transform calculus, Circ. Math. Palermo, Series II 6 (1957), 325-333.

12. J. Mikusiński, Sur les fondements du calcul opératoire, Studia Math. 11 (1949), 41-70.

13. Cz. Ryll-Nardzewski, Sur les séries de puissances dans le calcul opératoire, Studia Math. 13 (1953), 41-47.

14. A. Erdélyi, W. Magnus, F. Oberhettinger, F. Tricomi and Bateman staff, Higher transcendental functions, Vol. II, McGraw-Hill, New York, 1953.

15. — , Tables of integral transforms, Vol. I, McGraw-Hill, New York, 1954.

UNIVERSITY OF WASHINGTON,

SeATtLe, Washington 